\title{
Cement Augmented MIS Screw Vertebroplasty, Hybrid Procedure - A Technical Note
}

\section{Atif Naseem*, Ayush Sharma, Vijay Singh, Manoj B Gaddikeri, Harsh Agrawal, Nandan Marathe, Aakash Shakya and Pratik Sidhdhapuria}

Department of Orthopaedics, and Spine Surgery, Bharat Ratna Dr. Babasaheb

Ambedkar Memorial Hospital, Central Railway Byculla-Mumbai, India

*Corresponding Author: Atif Naseem, Department of Orthopaedics, and Spine Surgery, Bharat Ratna Dr. Babasaheb Ambedkar Memorial Hospital, Central Railway

Byculla-Mumbai, India
Received: October 19, 2021

Published: November 25, 2021

(C) All rights are reserved by Atif Naseem., et

al.

\begin{abstract}
Osteoporotic Vertebral compression fracture occurs in approximately $20 \%$ of individuals over 70 years of age. A significant majority of fractures approx $60 \%$ to $75 \%$ occur around the thoracolumbar region. The fractures can result in persistent pain, inability to perform daily activities, and a marked decrease in the quality of life. Reduction of pain and stabilization of vertebrae and prevention of deformity are the goals of treatment in vertebral compression fracture. The purpose of this technical note is to demonstrate the usefulness of minimal invasive cement augmented screw vertebroplasty along with short segment fixation at junctional vertebral compression fracture and its intraoperative effectiveness in preventing devastating complications.
\end{abstract}

Keywords: Vertebroplasty; Cement; PMMA

\section{Introduction}

Vertebroplasty was first performed in 1984 by Frenchradiologists for treatment of a hemangioma. Since then Vertebroplasty eventually went on to revolutionize the treatment of painful osteoporotic vertebral compression fractures and few vertebral body tumors. Nowadays vertebroplasty is mainly performed by orthopedics and spinesurgeons. Though there are very few complications in this procedure [1] like cement leakage in adjacent areas, however, sometimes PMMA (cement) venous embolization can be lifethreatening causing complications such as pulmonary embolism, acute respiratory failure secondary to respiratorydistress, intracranial embolic stroke, inferior vena cava syndrome and cardiac complication - which are noted in various studies. These lifethreatening complications are uncommon but can be easily prevented by thorough planning,mitigation and safe technique. The purpose of this technical note is to demonstrate the usefulness of minimal invasive screw vertebroplasty along with short segment fixation [2] at jjunctional vertebral compression fracture (VCF ) and its intraoperative effectiveness inpreventing devastating complications.

Osteoporosis is a systemic bone disorder characterized by a reduction in bone mass. Vertebral compression fracture occursin approximately $20 \%$ of individuals over 70 years of age.

The fractures can result in persistent pain, inability to perform daily activities, and a marked decrease in the quality of life.

Reduction of pain and stabilization of vertebrae are the goalsof treatment in vertebral compression fracture. Percutaneous vertebroplasty $[1,7]$ is a minimally invasive method involvinginjection of polymethylmethacrylate (PMMA) into the vertebral body to stabilize the fracture. Pain relief is usually observed within first 72 hours after treatment. There are concerns regarding procedural complications, which are mostly related to leakage of PMMA into adjacent structures, or injection into the vertebral venous plexus. 
Such venous leaks cause compression of the spinal cord or nerves or couldalso cause pulmonary embolism. The overall complication rate was reported to range from 1 to $10 \%$, with a higher incidence of complication in cases with the metastatic lesion.Several other surgical methods of treatment include balloon kyphoplasty, vertebroplasty combined with pedicle screw stabilization (hybrid procedure), cement augmented pedicle screw stabilization [5,6], decompression, and stabilization. Theaim of our study was to analyze the technical consideration of hybrid procedure combined with cement augmented pedicle screw and the prevention of complication arising out of traditional vertebroplasty surgery.

\section{Technique}

MIS screw vertebroplasty under image intensifier wasplanned in a 70-year female with D12 vertebral body osteoporotic wedge compression fracture.


Figure 1: Cobbs $40.1 \mathrm{deg}$, Local kyphotic angle - $30 \mathrm{deg}$.

Similar to routine vertebroplasty [7] the patient was positioned and the procedure was carried out under general anesthesia in prone position. Localization of the pedicles was performed with the aid of the c-arm fluoroscopy. We used the transpedicular route to reach the vertebral body. Pedicles provides a definite anatomical landmark for needle targeting and prevent damage to adjacent structures. Using the transpedicular route, the Jamshedi needle was centered at the 8 o'clock over the left pedicle or 4 o'clock over the right pedicle on the AP view. One may have to start the entry pointslightly more superior so that the needle is able to traverse thevertebrae without penetrating the fractured and collapsed superior end plate. The needle was medialized through thecylinder of the pedicle to reach the middle of the vertebra.

Once the first cortex was pierced and a footprint was obtained by the needle in the pedicle, and the position was considered ideal on the AP view, advancement of the needle was done under the guidance of the lateral fluoroscopy such that tip should not cross medial wall of pedicle.

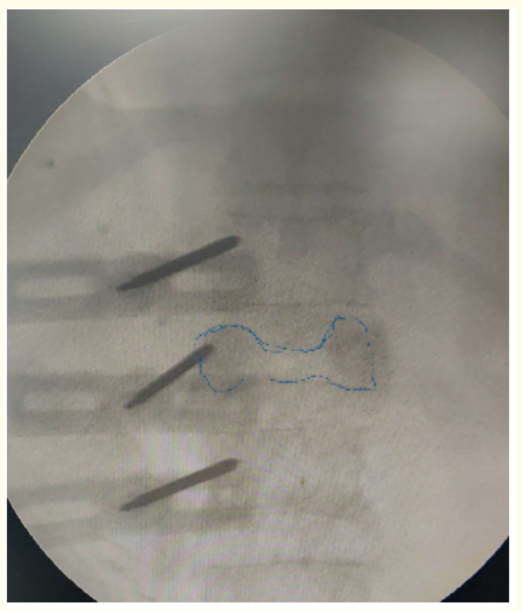

Figure 2

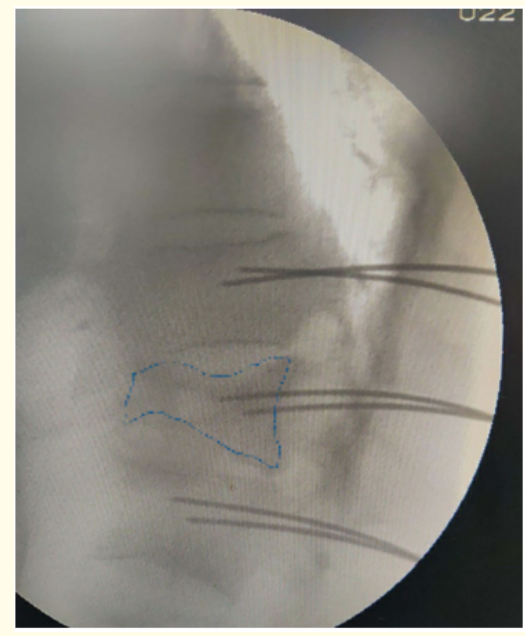

Figure 3 
Care should be taken in osteoporotic bone where penetrating the bony cortex and advancing the needle into the vertebral body is easy. The tip of the needle should lie beyond the midpoint of the vertebral body on the lateral view. The ideal endpoint was the junction between the anterior and middle thirds of the vertebral body.

In our practice of screw vertebroplasty Radioqpaque dye was not used, after crossing pedicle we removed the stellate and inserted guide wire into the vertebral body and then safely removed Jamshedi needle keeping guide wire secured. This procedure was done for D11-D12-L1 vertebrae [2] on one side and then repeated on contralateral side, this step is followed by dilatation tapping and screw placement of adequate length. At fractured level weused fenestrated cannulated dural thread polyaxial pedicular screw. Dural threads add more sturdy holds in pedicle whilerest of the screw are MIS cannulated screws.

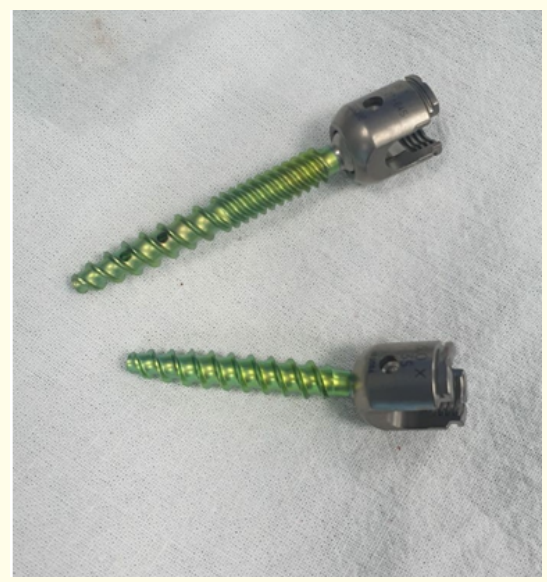

Figure 4

After screw placement cementing apparatus consist of sleeves and cement pusher which further add up to safety concerns [4] and preventing consequences of cement leakage. Preparation of cement was done with the help of mixing device once all screw had been placed followed by simultaneous cementing with the help of plunger. In our practice we also cement upperand lower vertebrae in osteoporotic spine.

The procedure is continuously monitored with fluoroscopy. We usually put $1-2 \mathrm{ml}$ of cement on either side, more in fractured vertebrae. After final placement of rod on both side closure done in layers.
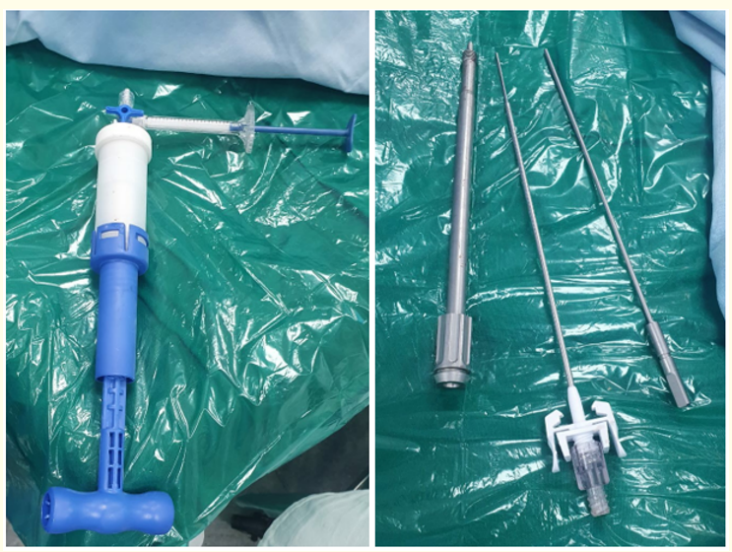

Figure 5: Depuy-synthes cement augment system.

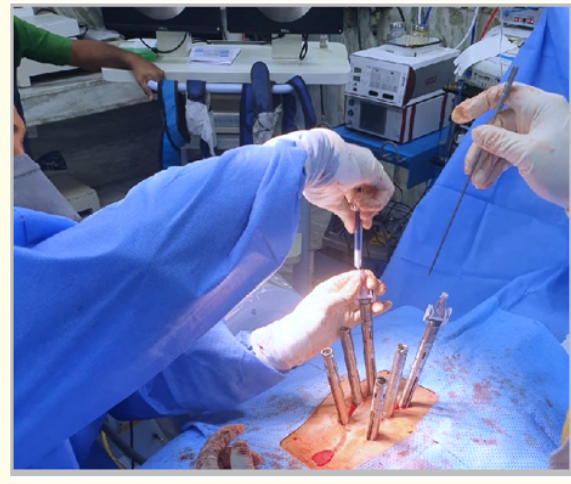

Figure 6 
bilization, percutaneous minimally invasive pedicle screw fixation and stabilization can be performed tominimize muscle injury and enable early rehabilitation. The application of posterior minimally invasive techniques reduces the approach-related morbidity like iatrogenic muscle denervation, ischemia, pain and functional impairment [11]. Augmentation of pedicle screws with PMMA is the most effective and practical technique to improve the hold of the screws in the bone.The combination of Kyphoplasty or Percutaneous vertebroplasty and instrumentation, so called hybrid stabilization, can effectively shorten the entire construct and therefore help to minimize the surgical trauma. Additionally, percutaneous instrumentation further decreases the invasiveness of the procedure [12]. Percutaneous Vertebroplasty alone appeared to provide better vertebral height restoration but could not improve segmental kyphosis or even thoracolumbar kyphosis leading to higher incidence of new Adjacent Segment Fractures (ASF's) compared to conservative treatment [13]. Screw vertebroplasty as a hybrid procedure at this level should be planned with aid of CT scan [9]. Hence there should not be any burst fracture or posterior wall damage or damage to pedicles, CT scan also help in assessment of pedicle morphology before we execute to final planning of screw vertebroplasty. DEXA Scan should be performed to conclude systemic osteoporosis. MRI will also aid in planning when it comes to neuro-deficit or posterior tension band damage or chance fracture. Therefore at junctional level through cement augmented screw vertebroplasty we could achieve several parameters like anterior reconstruction through cement, stabilizing posterior tension band via short segment fixation, correction of local kyphotic deformity and prophylaxis for prevention of futureadjacent segment Fractures in osteoporosis.
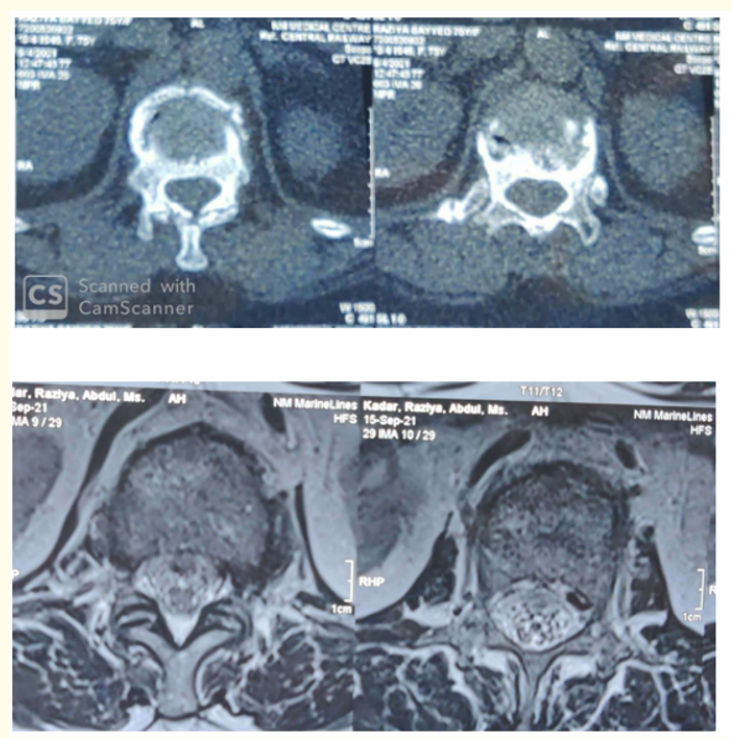

Figure 7

\section{Conclusion}

The procedure of cement augmented screw vertebroplasty with short segment fixation as a hybrid procedure through minimal invasive spine approach can really achieve milestones particularly at junctional level vertebral compression fractures. On view of safety concerns also, this technique reduces venous leakage of cement significantly, and risk of complications can be nullified.
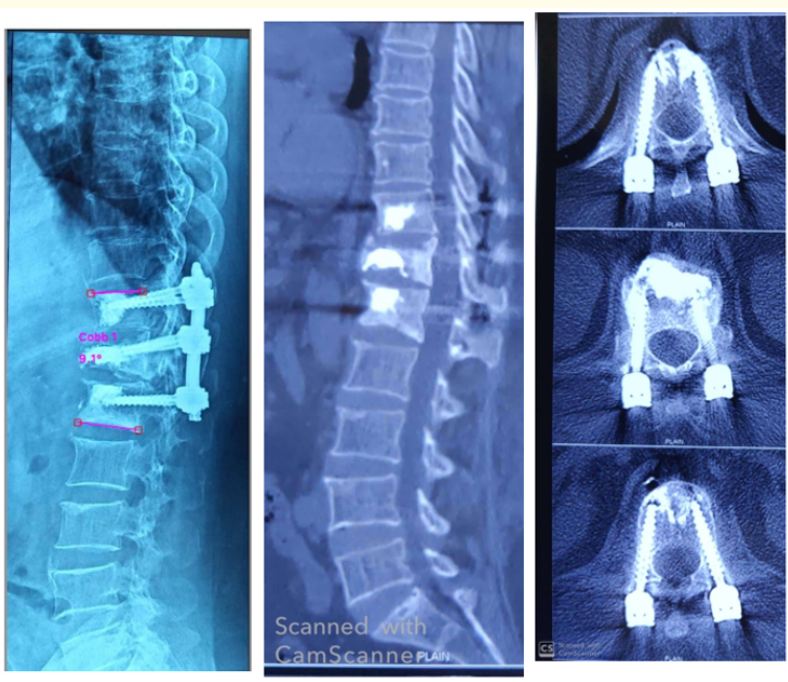

Figure 8

\section{Bibliography}

1. Pitton MB., et al. "CT- guided vertebroplasty: analysis of technical results, extraosseous cement leakages, and complications in 500 procedures". European Radiology 18.11 (2008): 2568-2578.

2. Rajsekaran S., et al. "Osteoporotic thoracolumbar fracturehow are they different? classification and treatment algorithm". Journal of Orthopaedic Trauma 31 (2017): S46.

3. Massimo Girardo., et al. "Surgical treatment of osteoporotic thoraco-lumbar compressive fractures: the use of pedicle screw with augmentation PMMA". European Spine Journal 26 (2017): 546-551.

4. Angela Coniglio., et al. "Effectiveness and reliability of cannulated fenestrated screws augmented with polymethylmethacrylate cement in the surgical treatment of osteoporotic vertebral fractures". Journal of Craniovertebral Junction and Spine 12.1 (2021): 33-37.

5. Vishwajeet Singh., et al. "Surgical Trend Analysis for Use of Cement Augmented Pedicle Screws in Osteoporosis of Spine: A Systematic Review (2000-2017)". Global Spine Journal 9.7 (2019): 783-795. 
6. Sébastien Pesenti., et al. "Percutaneous Cement-Augmented Screws Fixation in the Fractures of the Aging Spine: Is It the Solution?" BioMed Research International (2014).

7. Vincenzo Denaro., et al. "Vertebroplasty and kyphoplasty".

8. Rikin Hargunani., et al. "An Overview of Vertebroplasty: Current Status, Controversies, and Future Directions". Canadian Association of Radiologists Journal 63.2 (2012).

9. JT Mauch., et al. "Review of the Imaging Features of Benign Osteoporotic and Malignant Vertebral Compression Fractures". American Journal of Neuroradiology January (2018).

10. Daniela Alexandru and William So. "Evaluation and Management of Vertebral Compression Fractures". Perm Journal 16.4 (2012): 46-51.

11. S Rajasekaran., et al. "Management of thoracolumbar spine trauma: An overview".

12. Shanmuganathan Rajasekaran., et al. "Osteoporotic Thoracolumbar Fractures-How Are They Different?-Classification and Treatment Algorithm". Indian Journal of Orthopaedics 49.1 (2015): 72-82.

13. Xinjie Liang., et al. "Risk factors of adjacent segmental fractures when percutaneous vertebroplasty is performed for the treatment of osteoporotic thoracolumbar fractures".

Volume 4 Issue 12 December 2021

(C) All rights are reserved by Atif Naseem., et al. 